THP-6(T1)

\title{
Optical coherence tomography and optical time-domain reflectometry utilizing white light emitting diodes
}

\author{
Hui-Chuan Cheng and Chi-Kuang Sun \\ Graduate Institute of Electro-Optical Engineering and Department of Electrical Engineering, \\ National Taiwan University, Taipei 10617, TAIWAN \\ Phone : 886-2-23659703, FAX : 886-2-23677467, E-mail : chenghc@ntu.edu.tw
}

\begin{abstract}
Nitride based white-light-emitting-diodes (WLED) are utilized in high resolution OCT and OTDR systems. Submicron longitudinal resolution was demonstrated.
\end{abstract}

Optical coherence tomography (OCT) and optical time-domain reflectometry (OTDR) provide noninvasive morphological cross-sectional information on biological tissues and materials [1]. Short temporal coherence length (i.e., broadband) and high irradiance are required for OCT and OTDR sources. With a broadband light source, not only high spatial resolution but also spectral information can all be obtained, called spectroscopic OCT (SOCT) [2]. Several applicable sources are used nowadays, such as continuum generation in microstructure optical fibers, low-noise superluminescent diodes, and mode-locked lasers, etc. Besides these light sources, we find nitride based white-light-emitting-diodes (WLED) with broadband emission can provide sub-micron spatial resolution. Because the spectrum of WLED spreads in visible light, it could also provide excellent spectroscopic information especially for biological investigations [3].

Blue light LED based WLED was developed since 1996 by combining with newly developed high-efficiency YAG phosphor. Recently, different types of WLEDs applying various combinations of UV/blue LEDs and phosphors are also under development, and their performance can be expected to be better than $50 \mathrm{~lm} / \mathrm{W}$ by 2010 . With their compactness and durability (with a service life around 100,000 hours), WLEDs make maintenance an easy task With high energy efficient and inexpensiveness, WLED becomes an attractive light source for future OCT and OTDR applications. In this letter, we report our recent experimental results on the development of WLED-based OCT and OTDR systems. Sub-micron resolution in samples and $1 \mu \mathrm{m}$-resolution in air was easily achieved.

Different types of WLED provide different output spectra, thus with different spectral widths. Figure1 shows one of the WLED spectra with a $190 \mathrm{~nm}(\sim 200 \mathrm{THz})$ bandwidth at a $532 \mathrm{~nm}$ center wavelength. The blue part of the spectrum is contributed from the InGaN-LED while the yellow part of the spectrum is contributed from the phosphors. With such a broad emission bandwidth covering the whole visible region, a sub-micron spatial resolution should be expected. In Figure2, we shows the interference trace of this WLED and $1.0 \mu \mathrm{m}$ FWHM in the air is achieved. According to the Weiner-Khinchin theorem, we took the autocorration trace(Fig.2.) into the Fast Fourier Transform. We can get the auto-power spectrum which shows in Figure3. By comparing the Figure3 with the spectra in Figure1, which was measured by the grating spectrometer, we find these two spectra profiles fit well with each other. In Figurel there is a specific spike at $610 \mathrm{~nm}$, and this spike can also be resolved clearly in Figure3. Figure4 shows an example interference trace taken with the WLED-based OCT system in a $3.65 \mu \mathrm{m}$-thick GaN sample grown on a sapphire substrate. The WLED emission was collected into a single-mode fiber for mode shaping before used as the OCT light source. $370 \mathrm{~nm}$ longitudinal resolution in $\mathrm{GaN}$ was achived with a corresponding $1.0 \mu \mathrm{m}$ longitudal resolution in air (refractive index $n=2.7$ in $\mathrm{GaN}$ ). The first interference spike corresponds to 


\section{THP-6(T1)}

the light reflected on the air/GaN interface while the second interference spike occurs at the interface of $\mathrm{GaN}$ layer and sapphire substrate. The distance between peaks of these two interference patterns is $3.63 \mu \mathrm{m}$ in $\mathrm{GaN}(9.8 \mu \mathrm{m}$ in air). With a $370 \mathrm{~nm}$ spike-width, these two interfaces $(3.63 \mu \mathrm{m}$ apart) can thus be clearly resolved.

In conclusion we show the great potential in high resolution OCT and spectroscopic OCT (SOCT) utilizing WLED light sources. More detailed discussion including WLED based OCT and spectral study will be presented in the conference.

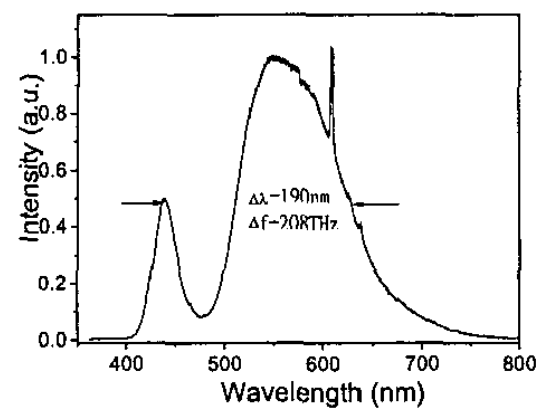

Fig.1. Output spectrum of a WLED.

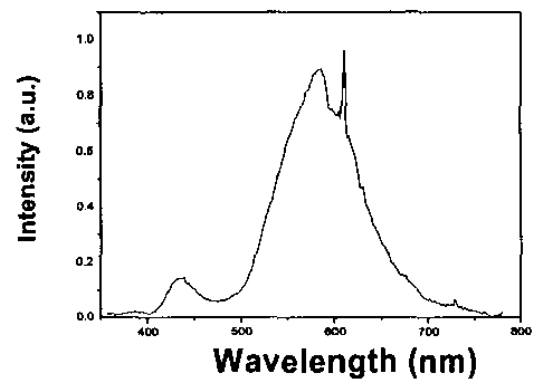

Fig.3. F.F.T. of Fig.2.

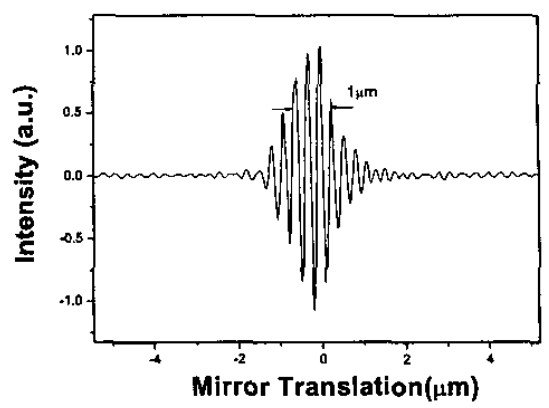

Fig.2. Interference pattern in the air.

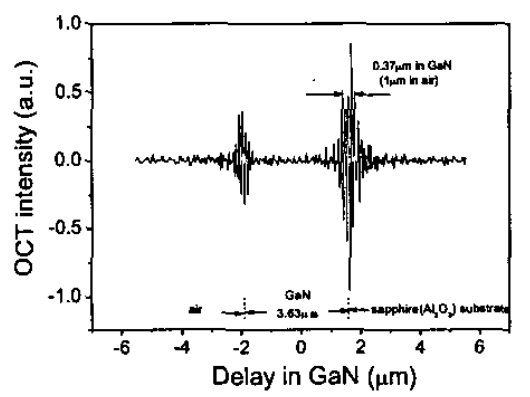

Fig.4. Interference pattern in a $\mathrm{GaN}$ thin film taken with the WLED-based ultra-high resolution OCT system.

\section{References}

[1] Joseph M. Schmitt,"Optical Coherence Tomography (OCT): A Review," IEEE journal of selected topics in quantum electronics,vol.5, no 4,July/August 1999.

[2] U. Morgner, W. Drexler, F. X. K"artner, X. D. Li, C. Pitris, E. P. Ippen, and J. G.

Fujimoto, "Spectroscopic optical coherence tomography," Optics letters, Vol. 25, No. 2, January 15,2000

[3] R.Leitgeb, "Spectral measurement of absorption by spectroscopic frequency-domain optical coherence tomography," Optics letters, Vol. 25, No. 11, June 1, 2000 